\title{
A Three-Dimensional Locally One-Dimensional Multiresolution Time-Domain Method Using Daubechies Scaling Function
}

\author{
Jaejong $\mathrm{Ryu}^{1} \cdot$ Wuseong $\mathrm{Lee}^{1} \cdot$ Hachul $\mathrm{Kim}^{2} \cdot$ Hyunchul Choi ${ }^{1}$
}

\begin{abstract}
A three-dimensional locally one-dimensional multiresolution time-domain(LOD-MRTD) method is introduced and unconditional stability is proved analytically. The updating formulations have fewer terms on the right-hand side than those of an alternating direction implicit MRTD(ADI-MRTD). The validation of the method is presented using the resonance frequency problem of an empty cavity. The reduction of the numerical dispersion technique is also combined with the proposed method. The numerical examples show that the combined method can improve the accuracy significantly.
\end{abstract}

Key words : Locally One-Dimensional Multiresolution Time-Domain(LOD-MRTD), Alternating Direction Implicit Multiresolution Time-Domain(ADI-MRTD), Numerical Dispersion, Artificial Anisotropy.

\section{Introduction}

The multiresolution time-domain(MRTD) method applying wavelets expansions in space has been developed for solving electromagnetic problems ${ }^{[1]}$. Its numerical dispersion errors are significantly improved in comparison with the finite-difference time-domain(FDTD) method $^{[2]}$. However, the Courant-Friedich-Levy(CFL) stability condition still remains. An alternating direction implicit MRTD(ADI-MRTD) ${ }^{[3],[4]}$ method was proposed in order to overcome the CFL restriction. Recently, the unconditionally stable locally one-dimensional(LOD) time stepping technique has been applied to the FDTD me-

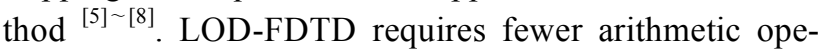
rations than ADI-FDTD while providing comparable accuracy.

As in the ADI method, the LOD method exhibits a splitting error that depends on not only the time step size, but also the spatial resolution. The time step is free of the CFL stability condition, but it cannot be made too large with the coarse mesh if a reasonable computational accuracy is to be achieved. To improve the accuracy, modified methods such as iterative techniques ${ }^{[9],[10]}$ and artificial anisotropy techniques ${ }^{[1] \sim[14]}$ were developed. The artificial anisotropy method takes only slightly more pre-computing than the conventional method, while the iterative method is computationally more expensive than the conventional method. In this paper, the LOD technique is applied to the MRTD, resulting in an unconditionally stable 3-D LOD-MRTD method. The algorithm based on Daubechies scaling functions is only considered for simplicity. Artificial anisotropy is also extended to the 3-D LOD-MRTD method to improve the accuracy. Enhanced numerical accuracy is shown in several numerical results.

\section{II . Formulations of 3-D LOD-MRTD Method}

The LOD-MRTD can be derived by applying the LOD algorithm to the MRTD schemes. The procedure is similar to the LOD-FDTD developed in [8]. The shifted Daubechies scaling functions with two vanishing moments $\left(\mathrm{D}_{2}\right)$ are considered for a simple representation of the LOD-MRTD formulation. Maxwell's curl equations in isotropic and lossless media are given as

$$
\nabla \times H=\varepsilon \frac{\partial E}{\partial t} \quad \nabla \times E=-\mu \frac{\partial H}{\partial t} .
$$

These equations can be rewritten in the Cartesian coordinates as

$$
\frac{\partial F}{\partial t}=[A] F+[B] F
$$

where $F=\left[E_{x}, E_{y}, E_{z}, H_{x}, H_{y}, H_{z}\right]^{T}$ and

Manuscript received September 2, 2009 ; revised November 23, 2009. (ID No. 20090902-037J)

${ }^{1}$ School of Electrical Engineering and Computer Science, Kyungpook National University, Daegu, Korea.

${ }^{2}$ Department of Information and Communication Engineering, Korea Naval Academy, Chinhae, Kyungnam, Korea. 


$$
\begin{aligned}
& {[A]=\left[\begin{array}{cccccc}
0 & 0 & 0 & 0 & 0 & \frac{\partial}{\varepsilon \partial y} \\
0 & 0 & 0 & \frac{\partial}{\varepsilon \partial z} & 0 & 0 \\
0 & 0 & 0 & 0 & \frac{\partial}{\varepsilon \partial x} & 0 \\
0 & \frac{\partial}{\mu \partial z} & 0 & 0 & 0 & 0 \\
0 & 0 & \frac{\partial}{\mu \partial x} & 0 & 0 & 0 \\
\frac{\partial}{\mu \partial y} & 0 & 0 & 0 & 0 & 0
\end{array}\right],} \\
& {[B]=\left[\begin{array}{cccccc}
0 & 0 & 0 & 0 & \frac{-\partial}{\varepsilon \partial z} & 0 \\
0 & 0 & 0 & 0 & 0 & \frac{-\partial}{\varepsilon \partial x} \\
0 & 0 & 0 & \frac{-\partial}{\varepsilon \partial y} & 0 & 0 \\
0 & 0 & \frac{-\partial}{\mu \partial y} & 0 & 0 & 0 \\
\frac{-\partial}{\mu \partial z} & 0 & 0 & 0 & 0 & 0 \\
0 & \frac{-\partial}{\mu \partial x} & 0 & 0 & 0 & 0
\end{array}\right],}
\end{aligned}
$$

in which $\varepsilon$ and $\mu$ present permittivity and permeability, respectively. The Crank-Nicolson $(\mathrm{CN})$ approximation is applied and then (2) can be split into the following two sub-steps

$$
\begin{aligned}
& \left(I-\frac{\Delta t}{2}[A]\right) F^{n+\frac{1}{2}}=\left(I+\frac{\Delta t}{2}[A]\right) F^{n} \\
& \left(I-\frac{\Delta t}{2}[B]\right) F^{n+1}=\left(I+\frac{\Delta t}{2}[B]\right) F^{n+\frac{1}{2}},
\end{aligned}
$$

where $I$ denotes the unit matrix. The Yee's grid is employed to discretize the field space, and the field quantities are only expanded in terms of the scaling function expansions. Therefore, the two sub-step procedures can be formulated as follows:

First sub-step from $n$ to $n+1 / 2$

Implicit updating for $E_{x}$

$$
\begin{aligned}
& { }_{x} E_{i+1 / 2, j, k}^{n+1 / 2}-\frac{(\Delta t)^{2}}{4 \mu \varepsilon(\Delta y)^{2}} \sum_{m=-3}^{2} a(m) \sum_{v=-3}^{2} a(v)_{x} E_{i+1 / 2, j+m+v+1, k}^{n+1 / 2} \\
& ={ }_{x} E_{i+1 / 2, j, k}^{n}+\frac{(\Delta t)^{2}}{4 \mu \varepsilon(\Delta y)^{2}} \sum_{m=-3}^{2} a(m) \sum_{v=-3}^{2} a(v)_{x} E_{i+1 / 2, j+m+v+1, k}^{n} \\
& +\frac{\Delta t}{\varepsilon \Delta y} \sum_{m=-3}^{2} a(m)_{z} H_{i+1 / 2, j+m+1 / 2, k}^{n}
\end{aligned}
$$

Explicit updating for $\mathrm{H}_{\mathrm{z}}$

$$
\begin{aligned}
& { }_{z} H_{i+1 / 2, j+1 / 2, k}^{n+1 / 2}={ }_{z} H_{i+1 / 2, j+1 / 2, k}^{n} \\
& +\frac{\Delta t}{2 \mu \Delta y} \sum_{v=-3}^{2} a(v)\left({ }_{x} E_{i+1 / 2, j+v+1, k}^{n+1 / 2}-{ }_{x} E_{i+1 / 2, j+v+1, k}^{n}\right)
\end{aligned}
$$

Second sub-step from $n+1 / 2$ to $n+1$ Implicit updating for $\mathrm{E}_{\mathrm{x}}$

$$
\begin{aligned}
& { }_{x} E_{i+1 / 2, j, k}^{n+1}-\frac{(\Delta t)^{2}}{4 \mu \varepsilon(\Delta z)^{2}} \sum_{m=-3}^{2} a(m) \sum_{v=-3}^{2} a(v)_{x} E_{i+1 / 2, j, k+m+v+1}^{n+1} \\
& ={ }_{x} E_{i+1 / 2, j, k}^{n+1 / 2}+\frac{(\Delta t)^{2}}{4 \mu \varepsilon(\Delta z)^{2}} \sum_{m=-3}^{2} a(m) \sum_{v=-3}^{2} a(v)_{x} E_{i+1 / 2, j, k+m+v+1}^{n+1 / 2} \\
& -\frac{\Delta t}{\varepsilon \Delta z} \sum_{m=-3}^{2} a(m)_{y} H_{i+1 / 2, j, k+m+1 / 2}^{n+1 / 2}
\end{aligned}
$$

$\underline{\text { Explicit updating for } \mathrm{H}_{\mathrm{y}}}$

$$
\begin{aligned}
{ }_{y} H_{i+1 / 2, j, k+1 / 2}^{n+1} & ={ }_{y} H_{i+1 / 2, j, k+1 / 2}^{n+1 / 2} \\
& -\frac{\Delta t}{2 \mu \Delta z} \sum_{v=-3}^{2} a(v)\left({ }_{x} E_{i+1 / 2, j, k+v+1}^{n+1}-{ }_{x} E_{i+1 / 2, j, k+v+1}^{n+1 / 2}\right),
\end{aligned}
$$

where the coefficients $a(v)$ (or $a(m))$ are the connection coefficients of the scaling function expansions. The coefficients $a(v)$ are reported in [4] and shown in Table 1. The values of $a(v)$ for $v<0$ are given by $a(-1-v)=-a(v)$. $\Delta x, \Delta y$ and $\Delta z$ represent the space discretization intervals in $x$-, $y$ - and $z$-direction, and $\Delta t$ is the time interval. The complete formulations for the unconditionally stable LOD-MRTD scheme can be obtained by applying the same procedure for other components. For derived formulations, there are six implicit and six explicit equations to be computed in one time step, which is the same number as those of the conventional ADI-MRTD method $^{[3]}$. However, as shown in $(4) \sim(7)$, the right-hand sides(RHS) of the equations have fewer terms than those in the ADI-MRTD method. The field component $E_{x}$, the second term of the RHS in (4), can be calculated at eleven adjacent grid points, while the field component is computed at 36 neighbor grid points in ADI-MRTD. Further, the introduced scheme needs only the summation of the field component $H_{z}$, the third term of RHS in (4), while the ADI-MRTD method needs the summations of both $H_{z}$ and $H_{y}$. Clearly, the LODMRTD computation is more concise than the ADIMRTD.

\section{Numerical Stability}

The equations in the spatial spectral domain can be written in the following matrix forms.

Table 1. Coefficient $a(v)$

\begin{tabular}{|c|c|}
\hline$v$ & $a(v)$ \\
\hline 0 & 1.22916661202745 \\
\hline 1 & -0.09374997764764 \\
\hline 2 & 0.01041666418309 \\
\hline
\end{tabular}


Sub-step 1: $F^{n+1 / 2}=\Lambda_{1} F^{n}$

Sub-step 2: $F^{n+1}=\Lambda_{2} F^{n+1 / 2}$,

where

$$
\Lambda_{1}=\left[\begin{array}{cccccc}
\frac{G_{y}}{Q_{y}} & 0 & 0 & 0 & 0 & \frac{-j 2 W_{y}}{\varepsilon Q_{y}} \\
0 & \frac{G_{z}}{Q_{z}} & 0 & \frac{-j 2 W_{z}}{\varepsilon Q_{z}} & 0 & 0 \\
0 & 0 & \frac{G_{x}}{Q_{x}} & 0 & \frac{-j 2 W_{x}}{\varepsilon Q_{x}} & 0 \\
0 & \frac{-j 2 W_{z}}{\mu Q_{z}} & 0 & \frac{G_{z}}{Q_{z}} & 0 & 0 \\
Q_{\alpha} & 0 & \frac{-j 2 W_{x}}{\mu Q_{x}} & 0 & \frac{G_{x}}{Q_{x}} & 0 \\
\frac{-j 2 W_{y}}{\mu Q_{y}} & 0 & 0 & 0 & 0 & \frac{G_{y}}{Q_{y}}
\end{array}\right]
$$

The above two step equations can be written as

$$
F^{n+1}=\Lambda_{2} \cdot \Lambda_{1} F^{n}=\Lambda F^{n} .
$$

The eigenvalues of the $\Lambda$ can be found as

$$
\begin{aligned}
& \lambda_{1}=\lambda_{2}=1 \\
& \lambda_{3}=\lambda_{4}=x+j y \\
& \lambda_{5}=\lambda_{6}=x-j y,
\end{aligned}
$$

where

$$
\begin{aligned}
& x=\frac{2\left(Q_{x}+Q_{y}+Q_{z}-Q_{x} Q_{y}-Q_{y} Q_{z}-Q_{z} Q_{x}\right)+Q_{x} Q_{y} Q_{z}}{Q_{x} Q_{y} Q_{z}} \\
& y=\sqrt{1-x^{2}} .
\end{aligned}
$$

It is easy to show that $\left|\lambda_{3}\right|=\left|\lambda_{4}\right|=\left|\lambda_{5}\right|=\left|\lambda_{6}\right|=1$. Therefore, the introduced LOD-MRTD method is uncondi- tionally stable.

\section{Numerical Examples}

For validation of the proposed LOD-MRTD method, an empty cavity with dimensions of $90 \times 60 \times 150 \mathrm{~mm}$ was computed. In the first case, the mesh chosen was rather dense. For a uniform standard Yee's cell, the spatial steps in all dimensions were chosen to be $\Delta \alpha=10$ mm with $\alpha=x, y$ and $z$, leading to a $9 \times 6 \times 15$ grid. The time step was varied from 0.2 to five times the CFL limit of the original scaling function based MRTD(SMRTD). The CFL time step limit of the original SMRTD with $\mathrm{D}_{2}$ is $0.433\left(\Delta \alpha / c_{0}\right)$, where $c_{0}$ is the speed of light in the vacuum. Electric fields are sampled for a period of $108.3 \mathrm{~ns}$. Namely, the iterations are assumed to be 5,000 when the relative time step is 0.5 . Fig. 1 shows the relative errors from the analytical solutions for the two resonant modes, $\mathrm{TE}_{101}$ and $\mathrm{TE}_{102}$. The horizontal axis is the ratio of the LOD-MRTD time step $\Delta t$ to the CFL time step limit $\left(\Delta t_{\mathrm{MRTD}-\mathrm{CFL}}=0.433\left(\Delta \alpha / c_{0}\right)\right)$. It can be seen that, when the time step increases to four times the CFL time step limit, the errors are still relatively small, less than $2.78 \%$ for the $\mathrm{TE}_{101}$ mode. In the second case, a coarse mesh was chosen with the space step being $30 \mathrm{~mm}$. It led to a $3 \times 2 \times 5$ grid. Fig. 2 shows the computation results. With a coarse mesh, the time step can no longer be too large. Even with the time step of twice the CFL time step limit, the error with the LOD-MRTD for the lowest resonant mode $\left(\mathrm{TE}_{101}\right)$ becomes lager than $5.61 \%$. When the relative time step is 0.5 , Table 2 shows the numerical results in terms of the mesh size, lowest resonance frequency, relative

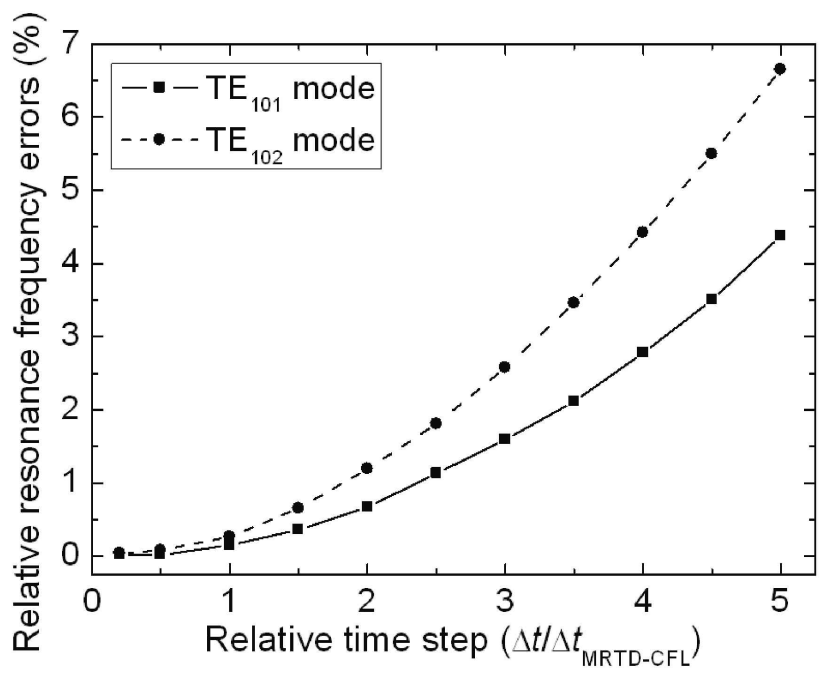

Fig. 1. Relative resonance frequency errors in the cavity $(9 \times 6 \times 15$ cells $)$ as a function of the relative time $\operatorname{step}\left(\mathrm{TE}_{101}: 1.942 \mathrm{GHz}, \mathrm{TE}_{102}: 2.602 \mathrm{GHz}\right)$. 


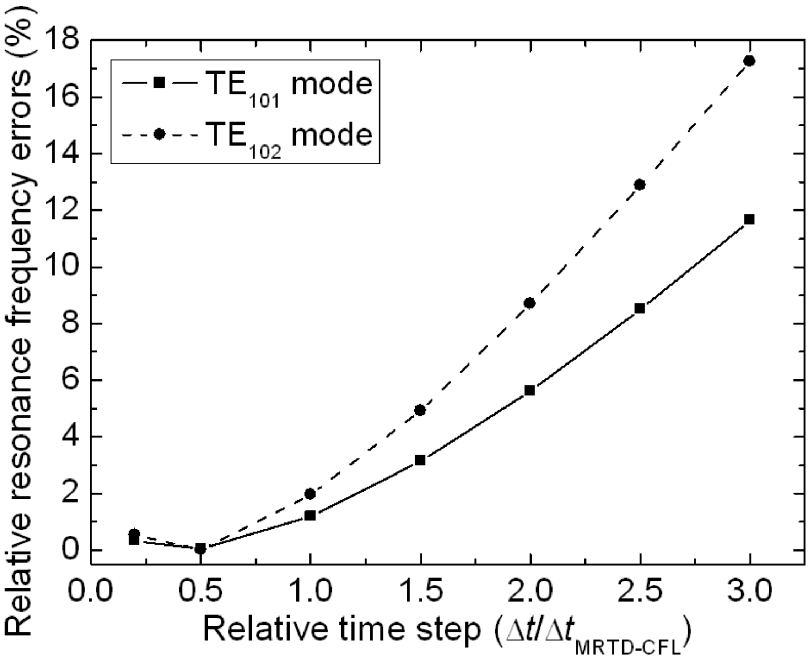

Fig. 2. Relative resonance frequency errors in the cavity $(3 \times 2 \times 5$ cells $)$ as a function of the relative time $\operatorname{step}\left(\mathrm{TE}_{101}: 1.942 \mathrm{GHz}, \mathrm{TE}_{102}: 2.602 \mathrm{GHz}\right)$.

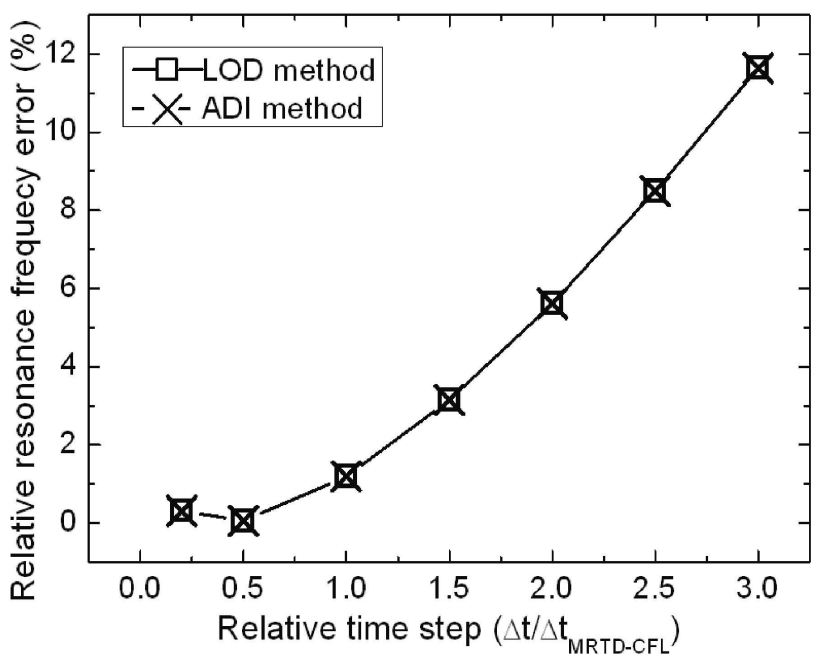

Fig. 3. Comparison of the relative resonance frequency errors in the cavity $(3 \times 2 \times 5$ cells $)$ for the lowest resonance frequency( $\left.\mathrm{TE}_{101}: 1.942 \mathrm{GHz}\right)$.

error, and computational time. Frequency is given in $\mathrm{GHz}$, error in \%, and time in seconds. These simulations are performed on Intel core 2 duo $(2.33 \mathrm{GHz})$ PC. As can be seen, the errors for both ADI- and LOD-MRTD are at the same level, but the computational time is less in LOD-MRTD. The FDTD method needs denser spatial intervals in order to obtain the accurate results. The relative errors of the LOD- MRTD and the ADI-MRTD from the analytical solutions, with respect to relative time steps, are shown in Fig. 3. It is found that both methods have the same relative errors. Although the time step is free of the CFL stability condition, it cannot be made too large in case of the coarse mesh.
Table 2. Comparison of numerical results.

\begin{tabular}{|c|c|c|c|c|c|c|}
\hline \multirow{3}{*}{$\begin{array}{c}\text { Mesh } \\
\text { (Iteration) }\end{array}$} & \multicolumn{2}{|c|}{ FDTD } & \multicolumn{2}{|c|}{ ADI-MRTD } & \multicolumn{2}{|c|}{ LOD-MRTD } \\
\hline & $\begin{array}{l}\text { Results } \\
\text { (GHz) }\end{array}$ & \multirow{2}{*}{$\begin{array}{l}\text { Time } \\
(\mathrm{sec})\end{array}$} & $\begin{array}{c}\text { Results } \\
(\mathrm{GHz})\end{array}$ & \multirow{2}{*}{$\begin{array}{l}\text { Time } \\
(\mathrm{sec})\end{array}$} & $\begin{array}{c}\text { Results } \\
\text { (GHz) }\end{array}$ & \multirow{2}{*}{$\begin{array}{l}\text { Time } \\
(\mathrm{sec})\end{array}$} \\
\hline & (Error) & & (Error) & & (Error) & \\
\hline \multirow{2}{*}{$\begin{array}{c}3 \times 2 \times 5 \\
(5,000)\end{array}$} & 1.875 & \multirow{2}{*}{3.25} & 1.941 & \multirow{2}{*}{46.76} & 1.941 & \multirow{2}{*}{16.98} \\
\hline & $(3.40 \%)$ & & $(0.05 \%)$ & & $(0.05 \%)$ & \\
\hline \multirow{2}{*}{$\begin{array}{c}6 \times 4 \times 10 \\
(10,000)\end{array}$} & 1.925 & \multirow{2}{*}{13.82} & & & & \\
\hline & $(0.82 \%)$ & & & & & \\
\hline \multirow{2}{*}{$\begin{array}{c}9 \times 6 \times 15 \\
(15,000)\end{array}$} & 1.935 & \multirow{2}{*}{36.70} & & & & \\
\hline & $(0.31 \%)$ & & & & & \\
\hline
\end{tabular}

\section{Numerical Dispersion and Optimization}

The numerical dispersion relation can be established using a procedure similar to the one described in [15]. Equation (9) is rewritten in the spectral domain as

$$
\left(e^{j \omega \Delta t} I-\Lambda\right) F=0
$$

The resulting numerical dispersion relation for freespace is

$$
\tan ^{2}\left(\frac{\omega \Delta t}{2}\right)=\frac{P_{x}+P_{y}+P_{z}+P_{x} P_{y}+P_{y} P_{z}+P_{z} P_{x}}{1+P_{x} P_{y} P_{z}},
$$

where

$$
P_{\alpha}=\frac{W_{\alpha}^{2}}{\mu_{0} \varepsilon_{0}}, \quad \alpha=x, y, z .
$$

In order to reduce the dispersion error, the numerical dispersion relation in magnetically and electrically anisotropic material can be considered. Assume that the relative permeability is equal to the relative permittivity (i.e., $\left.\varepsilon_{r}=\operatorname{diag}\left(\varepsilon_{x}, \varepsilon_{y}, \varepsilon_{z}\right)=\mu_{r}\right)$. The numerical dispersion relation can be obtained as the following form in free space.

$$
\begin{aligned}
\tan ^{2}\left(\frac{\omega \Delta t}{2}\right) & =\left[\frac{P_{x}}{\varepsilon_{y} \varepsilon_{z}}+\frac{P_{y}}{\varepsilon_{z} \varepsilon_{x}}+\frac{P_{z}}{\varepsilon_{x} \varepsilon_{y}}+\frac{P_{x} P_{y}}{\varepsilon_{x} \varepsilon_{y} \varepsilon_{z}{ }^{2}}\right. \\
& \left.+\frac{P_{y} P_{z}}{\varepsilon_{x}{ }^{2} \varepsilon_{y} \varepsilon_{z}}+\frac{P_{z} P_{x}}{\varepsilon_{x} \varepsilon_{y}{ }^{2} \varepsilon_{z}}\right] /\left[1+\frac{P_{x} P_{y} P_{z}}{\varepsilon_{x}{ }^{2} \varepsilon_{y}{ }^{2} \varepsilon_{z}{ }^{2}}\right]
\end{aligned}
$$

It is exactly the same as the relation of the ADIMRTD method. Therefore, the reduction procedure for the numerical dispersion error can be employed in the LOD-MRTD method, as it previously worked for the ADI-MRTD method ${ }^{[14]}$. As in ADI-MRTD, a Courant coefficient $S$ and the spatial resolution coefficient $R$ are defined as 


$$
\begin{aligned}
& S=c_{0} \Delta t \sqrt{\frac{1}{\varepsilon_{y} \varepsilon_{z} \Delta x^{2}}+\frac{1}{\varepsilon_{z} \varepsilon_{x} \Delta y^{2}}+\frac{1}{\varepsilon_{x} \varepsilon_{y} \Delta z^{2}}} \\
& R=\frac{\lambda}{\sqrt{\Delta x^{2}+\Delta y^{2}+\Delta z^{2}}} .
\end{aligned}
$$

The parameters are introduced to denote the shape of a single Yee's cell.

$$
Z_{1}=\frac{\Delta x}{\Delta y}, \quad Z_{2}=\frac{\Delta x}{\Delta z}, \quad Z=\sqrt{1+Z_{1}^{-2}+Z_{2}^{-2}},
$$

where $\lambda=c_{0} / f$ is the wavelength and $c_{0}$ is the speed of light in the vacuum. If it is supposed that the wave propagation at angles $\phi$ and $\theta$ is in the spherical coordinate system, then $\bar{k}_{x}=k \sin \theta \cos \phi, \quad k_{y}=k \sin \theta \sin \phi, \quad k_{z}=$ $k \cos \theta$, and $k$ is denoted as $2 \pi / \widetilde{\lambda}$, where $\tilde{\lambda}$ is the wavelength in the grid. A coefficient $A=\left(v_{p} / c_{0}\right)=(\widetilde{\lambda} / \lambda)$ is defined as the normalized numerical phase velocity, where $v_{p}$ is the propagation velocity in the grid. In order to find the optimization parameter values for $\varepsilon_{x}, \varepsilon_{y}$, and $\varepsilon_{z}$ that ensure the minimum numerical dispersion, the values are first determined to yield $A=1$ along the coordinate directions $\left(\phi=0^{\circ}, \theta=90^{\circ}\right),\left(\phi=90^{\circ}, \theta=90^{\circ}\right)$, and $\left(\Theta=0^{\circ}\right)$. The optimization parameter values for $\varepsilon_{x}, \varepsilon_{y}$, and $\varepsilon_{z}$ are calculated as follows:

$$
\begin{aligned}
& \varepsilon_{x}=\frac{R Z Z_{1} Z_{2} L}{\pi S} \sqrt{\left(\frac{U_{2} U_{3}}{U_{1}}\right)} \cdot \tan ^{-1}\left(\frac{S}{L}\right) \\
& \varepsilon_{y}=\frac{R Z Z_{2} L}{\pi S Z_{1}} \sqrt{\left(\frac{U_{1} U_{3}}{U_{2}}\right)} \cdot \tan ^{-1}\left(\frac{S}{L}\right) \\
& \varepsilon_{z}=\frac{R Z Z_{1} L}{\pi S Z_{2}} \sqrt{\left(\frac{U_{1} U_{2}}{U_{3}}\right)} \cdot \tan ^{-1}\left(\frac{S}{L}\right),
\end{aligned}
$$

where

$$
\begin{aligned}
& U_{1}=\left\{\sum_{m=0}^{2} a(m) \sin \left[\frac{\pi(2 m+1)}{A R Z}\right]\right\}^{2} \\
& U_{2}=\left\{\sum_{m=0}^{2} a(m) \sin \left[\frac{\pi(2 m+1)}{A R Z Z_{1}}\right]\right\}^{2} \\
& U_{3}=\left\{\sum_{m=0}^{2} a(m) \sin \left[\frac{\pi(2 m+1)}{A R Z Z_{2}}\right]\right\}^{2} \\
& L=\sqrt{U_{1}^{-1}+U_{2}^{-1}+U_{3}^{-1}}
\end{aligned}
$$

After the calculated optimization parameters are applied to update the schemes, the relative numerical phase velocity $A$ is 1 along the axial directions. The phase velocity anisotropic error is obtained as

$$
\Delta \%_{\text {aniso }}=\frac{\max \left(v_{p}(\phi, \theta)\right)-\min \left(v_{p}(\phi, \theta)\right)}{c_{0}} .
$$

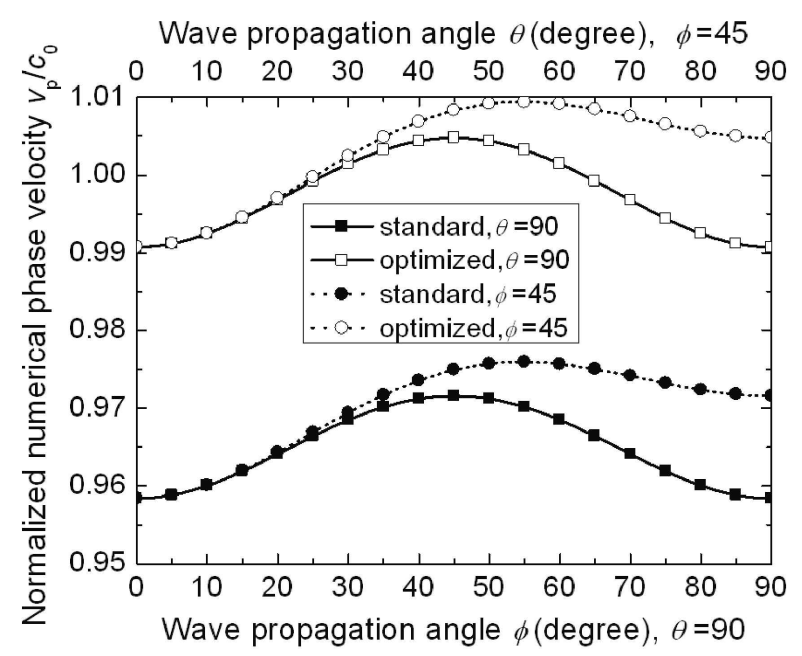

Fig. 4. Normalized phase velocity versus wave angles for standard and optimized LOD-MRTD.

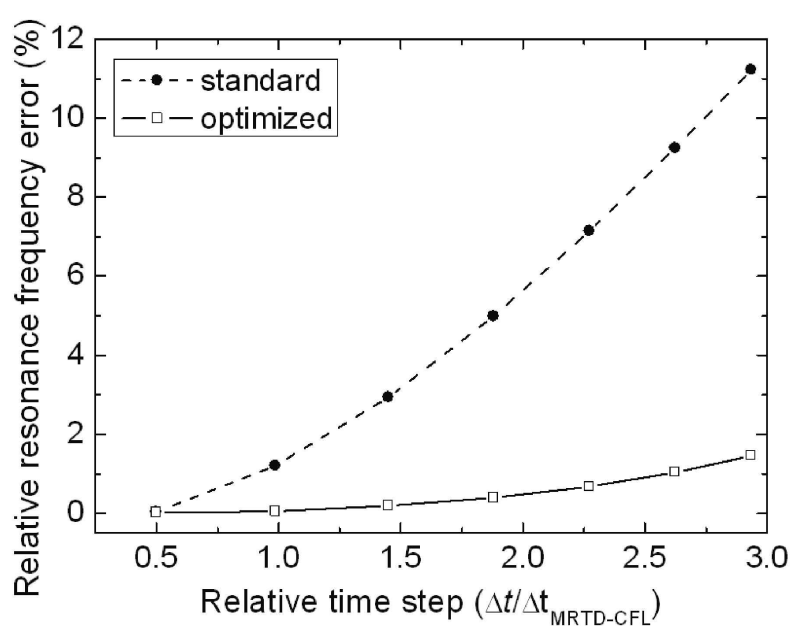

Fig. 5. Relative resonance frequency errors in the cavity $(90 \times 60 \times 150 \mathrm{~mm})$ for standard and optimized LOD$\operatorname{MRTD}\left(\mathrm{TE}_{101}: 1.942 \mathrm{GHz}\right)$.

When $\min \left(v_{p}(\phi, \Theta)\right)$ is equal to 1 , it is possible to obtain the optimization parameters by setting $A=1-\Delta \widetilde{V}$ aniso/2. On the other hand, setting $A=1+\Delta \tilde{V}_{\text {aniso }} / 2$ yields the optimization parameters, while $\max \left(v_{p}(\phi, \Theta)\right)$ is equal to 1 . Under this optimization, the numerical phase velocity errors have their minimum values in all propagation directions. Fig. 4 shows the effect of the correction with wave propagation angle. The horizontal top axis is the wave propagation angle $\theta$, while the angle $\phi$ is $45^{\circ}$. When the angle $\theta$ is $90^{\circ}$, the horizontal bottom axis is the wave propagation angle $\phi$. A spatial resolution coefficient $R=2.9709$ and the Courant coefficient $S=1.125\left(\Delta t \approx 1.45 \Delta t_{\mathrm{MRTD}-\mathrm{CFL}}\right)$ are considered. The parameters are optimized at $1.942 \mathrm{GHz}$. The calculated correction parameters are $\varepsilon_{x}=\varepsilon_{y}=\varepsilon_{z}=0.9667$ for the uniform grid. The maximum standard dispersion error is $-4.16 \%$, whereas the corrected dispersion error 
Table 3. Numerical results with different $\Delta t$.

\begin{tabular}{|c|c|c|c|c|c|c|}
\hline \multirow{4}{*}{$\begin{array}{c}\text { Analytical } \\
\text { result } \\
(\mathrm{GHz})\end{array}$} & \multicolumn{6}{|c|}{ The optimized LOD-MRTD scheme } \\
\hline & \multicolumn{2}{|c|}{$\begin{array}{c}\Delta t=0.987 \\
\Delta t_{\mathrm{MRTD}-\mathrm{CFL}}\end{array}$} & \multicolumn{2}{|c|}{$\begin{array}{c}\Delta t=1.88 \\
\Delta t_{\mathrm{MRTD}-\mathrm{CFL}}\end{array}$} & \multicolumn{2}{|c|}{$\begin{array}{c}\Delta t=2.62 \\
\Delta t_{\mathrm{MRTD}-\mathrm{CFL}}\end{array}$} \\
\hline & $\begin{array}{l}\text { Results } \\
\text { (GHz) }\end{array}$ & Time & $\begin{array}{c}\text { Results } \\
(\mathrm{GHz})\end{array}$ & Time & $\begin{array}{c}\text { Results } \\
(\mathrm{GHz})\end{array}$ & Time \\
\hline & (Error) & & (Error) & & (Error) & \\
\hline \multirow{2}{*}{1.942} & 1.943 & \multirow{2}{*}{8.61} & 1.950 & \multirow{2}{*}{4.55} & 1.962 & \multirow{2}{*}{3.30} \\
\hline & $(0.05 \%)$ & & $(0.41 \%)$ & & $(1.03 \%)$ & \\
\hline
\end{tabular}

Table 4. Comparison of results with standard and optimized LOD-MRTD.

\begin{tabular}{|c|c|c|c|c|}
\hline \multirow{2}{*}{$\begin{array}{c}\Delta t / \Delta t_{\mathrm{MRTD}} \\
\quad-\mathrm{CFL}\end{array}$} & \multicolumn{2}{|c|}{ Standard LOD-MRTD } & \multicolumn{2}{|c|}{ Optimized LOD-MRTD } \\
\hline & $\begin{array}{c}\text { Simulation } \\
\text { results(MHz) }\end{array}$ & $\begin{array}{c}\begin{array}{c}\text { Relative } \\
\text { error }\end{array}\end{array}$ & $\begin{array}{c}\text { Simulation } \\
\text { results(MHz) }\end{array}$ & $\begin{array}{c}\text { Relative } \\
\text { error }\end{array}$ \\
\hline 0.985 & 123.27 & $1.38 \%$ & 125 & $0.04 \%$ \\
\hline 1.4433 & 121.0 & $3.19 \%$ & 125. & $0.34 \%$ \\
\hline 1.8644 & 118.36 & $5.31 \%$ & 125.95 & $0.76 \%$ \\
\hline 2.2438 & 115.61 & $7.51 \%$ & 126.59 & $1.27 \%$ \\
\hline
\end{tabular}

remains within $\pm 1 \%$. The cavity with dimensions $90 \times 60$ $\times 150 \mathrm{~mm}$ is also computed with the optimization. The uniform spatial step $\Delta \alpha$ is chosen to be $30 \mathrm{~mm}$, which leads to $3 \times 2 \times 5$ cells. The correction parameters are optimized to wavelength $\lambda=0.1544 \mathrm{~m}(R=2.9709)$ at each time step. Fig. 5 illustrates the relative resonance frequency errors for the standard and corrected methods. When the relative time step is 2.62 , the error becomes $1.03 \%$ after the correction procedure, while the error without correction becomes larger than $9.2 \%$. Table 3 shows the lowest resonance frequency, relative error, and computational time in the optimized scheme. Their physical time periods are the same, but the number of iterations is different. When the time steps $\Delta t=0.987 \Delta t$ $\mathrm{MRTD}-\mathrm{CFL}=4.277 \times 10^{-11} \mathrm{~s}, \Delta t=1.88 \Delta t_{\mathrm{MRTD}-\mathrm{CFL}}=8.147 \times 10^{-11}$ $\mathrm{s}$, and $\Delta t=2.62 \Delta t_{\mathrm{MRTD}-\mathrm{CFL}}=1.137 \times 10^{-10} \mathrm{~s}$ are chosen, the iterations are 2532, 1329, and 952, respectively. Table 4 shows the lowest relative resonance frequency errors in the cavity with $1.5 \times 1 \times 2 \mathrm{~m}$. The uniform spatial step $\Delta \alpha$ is chosen to be $0.5 \mathrm{~m}$, which leads to $3 \times 2 \times 4$ cells. The correction parameters are optimized to an analytical resonance frequency of $125 \mathrm{MHz}(R=2.769)$ at each time step. It is obvious that using the combined method can improve the accuracy.

\section{Conclusion}

The unconditionally stable 3-D MRTD scheme based on the LOD method is introduced. Stability restriction associated with the conventional S-MRTD method is completely removed. The numerical errors of this method are same as those of the ADI-MRTD method. However, it requires less computational expenditure than the ADI-MRTD method because the updating equations have fewer terms on their right-hand sides. Moreover, this paper shows that the introduced work can be combined with an artificial anisotropy technique in order to reduce the numerical dispersion error. The combined method shows more accurate numerical results, even though the time step increases to several times of CFL limit of S-MRTD. Therefore the introduced LOD- MRTD method combined with dispersion reduction procedure can yield more efficient numerical computation for solving electromagnetic field problems.

This work was supported by the Grant of the Korean Ministry of Education, Science and Technology (The Regional Core Research Program/Antiaging and Well-being Research Center)

\section{References}

[1] M. Krumpholz, L. P. B. Katehi, "MRTD: New time domain scheme based on multiresolution analysis", IEEE Trans. Microw. Theory Tech., vol. 44, no. 2, pp. 555-571, Apr. 1996.

[2] K. S. Yee, "Numerical solution of initial boundary value problems involving Maxwell's equations in isotropic media", IEEE Trans. Ant. Propag., vol. AP14, no. 3, pp. 302-307, Apr. 1996.

[3] Z. Chen, J. Zhang, "Unconditionally stable 3-D ADI-MRTD method free of the CFL stability condition", IEEE Microw. Wireless Component Lett., vol. 11, no. 8, pp. 349-351, Aug. 2001.

[4] Y. Jing, J. Jilong, and S. Qiang, "Analysis of alternating direction implicit multiresolution time-domain method using Daubechies' scaling function", Proc. IEEE Int'l. Conf. Microw. Millimeter Wave Technol., vol. 2, pp. 663-665, 2008.

[5] J. Shibayama, M. Muraki, J. Yamauchi and H. Nakano, "Efficient implicit FDTD algorithm based on locally one-dimensional scheme", Electronics Letters, vol. 41, no. 19, pp. 1046-1047, Sep. 2005.

[6] E. L. Tan, "Unconditionally stable LOD-FDTD method for 3-D Maxwell's equations", IEEE Microw. Wireless Component Lett., vol. 17, no. 2, pp. 85-87, Feb. 2007.

[7] I. Ahmed, E. Chua, E. Li, and Z. Chen, "Development of the three-dimensional unconditionally stable LOD-FDTD method", IEEE Trans. Ant. Propag., 
vol. 56, no. 11, pp. 3596-3600, Nov. 2008.

[8] Q. Liu, Z. Chen, and W. Yan, "An efficient unconditionally stable three-dimensional LOD-FDTD method", IEEE MTT-S Int'l. Microw. Symp., pp. 45-48, Jun. 2008.

[9] K. Jung, F. L. Teixeira, "An iterative unconditionally stable LOD-FDTD", IEEE Microw. Wireless Component Lett., vol. 18, no. 2, pp. 76-78, Feb. 2008.

[10] K. Kong, M. Jeong, H. Lee, and S. Park, "A simple method to reduce the splitting error in the LODFDTD method", J. KIEES, vol. 9, no. 1, pp. 12-16, Mar. 2009.

[11] J. S. Juntunen, T. D. Tsiboukis, "Reduction of numerical dispersion in FDTD method through artificial anisotropy", IEEE Trans. Microw. Theory Tech., vol. 48, no. 4, pp. 582-588, Apr. 2000.

[12] H. Zheng, K. Leung, "An efficient method to re-

\section{Jaejong Ryu}

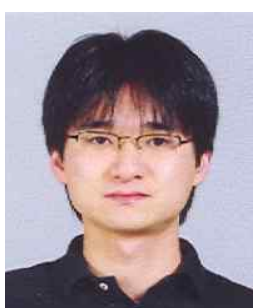

received a B.S. and M.S in the School of Electronic and Electrical Engineering from Kyungpook National University, Daegu, Korea, in 2002 and 2004, respectively. He is Ph.D. candidate in Electronic Engineering from Kyungpook National University. His current interests include the design of $\mathrm{RF}$ and microwave systems and computational electromagnetics.

Wuseong Lee

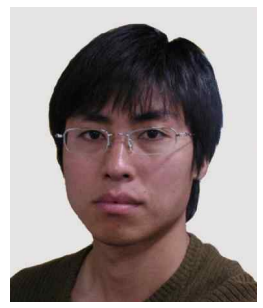

received a B.S. and M.S in the School of Electronic and Electrical Engineering and Computer Science from Kyungpook National University, Daegu, Korea, in 2003 and 2005, respectively, and is currently pursuing a Ph.D. degree in the School of Electrical Engineering and Computer Science from Kyungpook National University. His current interests include the design of RF systems, RF switches and filters. duce the numerical dispersion in the ADI-FDTD", IEEE Trans. Microw. Theory Tech., vol. 53, no. 7, pp. 2295-2301, Jul. 2005.

[13] A. P. Zhao, "Improvement on the numerical dispersion of 2-D ADI-FDTD with artificial anisotropy", IEEE Microw. Wireless Component Lett., vol. 14, no. 6, pp. 292-294, Jun. 2004.

[14] J. Ryu, W. Lee, J. Lee, Y. Moon, H. Kim, and H. Choi, "Reduction of numerical dispersion error in 3-D ADI-MRTD method with the coarse mesh", IEEE Microw. Wireless Component Lett., vol. 19, no. 8, pp. 485-487, Aug. 2009.

[15] F. Zheng, Z. Chen, "Numerical dispersion analysis of the unconditionally stable 3-D ADI-FDTD method", IEEE Trans. Microw. Theory Tech., vol. 49, no. 5, pp. 1006-1009, May 2001.

\section{Hachul Kim}

received his B.S. degree in Electronic Engineering in 1985

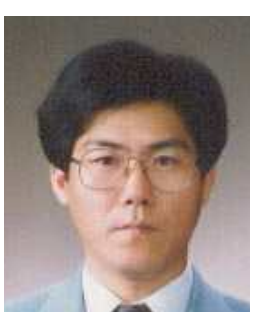

radar systems. and his M.S. and Ph.D. in Electronic Engineering from Kyungpook National University, Daegu, Korea, in 1990 and 1999, respectively. Since 1993, he has been a Professor in the Department of Information and Communication Engineering, Korea Naval Academy. His research interests include computational electromagnetics and

Hyunchul Choi

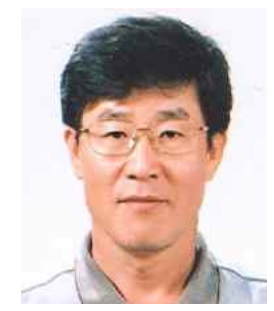

received his B.S. degree in Electronic Engineering from Kyungpook National University(KNU) in 1982, and M.S. and Ph.D. degrees in Electrical Engineering from the Korea Advanced Institute of Science and Technology(KAIST) in 1984 and 1989, respectively. His doctoral research concerned electromagnetic wave propagation and inverse scattering. From 1989 to 1990, he was with Daehyun Precision. Since 1990, he has been Professor at the School of Electronic and Electrical Engineering and Computer Science, KNU. His current work involves RF and microwave devices and computational electromagnetics. 